\title{
Determination of lipid composition and thermal phase transition temperature in an enriched plasma membrane fraction from ram spermatozoa
}

\author{
W. V. Holt and R. D. North \\ Gamete Biology Unit, Institute of Zoology, Zoological Society of London, Regent's Park, \\ London NWI 4RY, U.K.
}

\begin{abstract}
Summary. The preparation of a purified fraction of ram sperm plasma membranes is described and validated in this paper. Lipid analyses were performed on both the membrane preparation and whole spermatozoa; the main differences were that plasma membranes showed a significantly higher cholesterol :phospholipid molar ratio and a higher sphingomyelin content than did whole spermatozoa, but a lower proportional content of phosphatidylethanolamine. Enzymic assays revealed the presence of two distinct adenosine triphosphatases (ATPases) in the membrane fraction, activated independently by calcium and sodium ions. Arrhenius plots of the calcium-stimulated ATPase activity demonstrated that a change in energy of activation occurred in the region of $23^{\circ} \mathrm{C}$; it is believed that this is evidence for the occurrence of a thermal phase transition in the lipid environment of the enzyme molecules.
\end{abstract}

\section{Introduction}

Comparative studies into methods of semen preservation by chilling and freezing in the presence of cryoprotective substances have shown that the success of these procedures is markedly species dependent. Therefore, whilst the cryopreservation of bovine spermatozoa has become a routine process on a commercial scale, similar exploitation of semen preservation techniques has not been possible with other domestic species.

The reasons that determine this differential susceptibility of spermatozoa to cooling and freezing are poorly understood, but White \& Darin-Bennett (1976) and Darin-Bennett \& White (1977) suggested that differences in the sperm-lipid content were in some way related to the extent of coldshock damage sustained by spermatozoa. In particular, it was proposed that two ratios, cholesterol :phospholipid and unsaturated : saturated fatty acids, were important determinants. Empirically it was observed that spermatozoa with approximately equal proportions of cholesterol and phospholipid, for example, human and monkey, were less susceptible to cold shock than those containing proportionately lower amounts of cholesterol, i.e. bull, ram and boar. Similarly, spermatozoa from species in which the unsaturated : saturated fatty acid ratio was approximately $3: 1$, i.e bull, ram and boar, were more susceptible to cold shock than those of man or monkey, in which the equivalent ratio was nearer to unity.

As most sperm lipids are incorporated into various types of membrane structures, these results suggest that differences in membrane lipid composition between species are probably responsible for the differential labilities during storage. For example, the variations in lipid composition would probably lead to diversity in biophysical properties such as membrane fluidity.

More detailed investigation of the possible relationships outlined above, together with the application of biophysical probes of sperm plasma membrane properties, has been hampered by the 
difficulties encountered in the isolation of particular membrane fractions. A relatively simple procedure for the removal and isolation of sufficiently large quantities of membrane fraction to permit experimentation and biochemical analysis has been developed in this laboratory. Although conventional techniques have been employed, it would seem that contamination by acrosomal membrane is minimal; the plasma membrane-rich fractions thus obtained are therefore suitable material upon which to perform many studies. The validation of this technique, mainly by the use of stereometric analysis, is therefore presented in this paper together with some data on enzymic activities asssociated with the membrane fraction.

To investigate more closely the biochemistry of the plasma membrane, data on its lipid composition are also presented in this paper, together with similar data obtained for comparison from the analysis of whole spermatozoa. Preliminary experiments to establish the nature of the thermal phase transitions exhibited by the isolated membrane fraction are also reported. Biophysical characteristics of this type are known to be dependent upon membrane lipid content (for review, see Quinn, 1981) and may also be determining factors in the susceptibility of spermatozoa to coldinduced damage.

\section{Materials and Methods}

\section{Membrane preparation}

Semen was obtained from Finnish-Landrace rams by the use of an artificial vagina, and the ejaculates from 3 or 4 animals were pooled for the preparation of membranes.

After pooling, the semen (approximately $4 \mathrm{ml}$ ) was divided into four equal aliquots and washed through ficoll columns to remove the seminal plasma and most of the cytoplasmic droplets, using the method described by Harrison (1976). The washed spermatozoa were then resuspended in a Hepes-sucrose buffer, pH 7.4 (Hepes $20 \mathrm{mM}$, glucose $10 \mathrm{mM}, \mathrm{Na}_{2} \mathrm{HPO}_{4} 2 \mathrm{mM}, \mathrm{MgCl}_{2} 4 \mathrm{mM}, \mathrm{KOH}$ $2.5 \mathrm{~m}, \mathrm{NaOH} 2.5 \mathrm{mM}$, sucrose $200 \mathrm{mM}, \mathrm{NaCl} 14 \mathrm{mM}$, bovine serum albumin $1 \mathrm{mg} / \mathrm{ml}$ ), and centrifuged a second time $(500 \mathrm{~g}, 10 \mathrm{~min})$. Before the second centrifugation step $250 \mu \mathrm{g}$ trypsin inhibitor (soybean, type i-s, Sigma Chemical Company, Poole, U.K.) was added to each aliquot, as $100 \mu 1$ of a $2.5 \mathrm{mg} / \mathrm{ml}$ solution in water.

After the second centrifugation the spermatozoa were pooled once more in a total volume of about $6 \mathrm{ml}$. A further $250 \mu \mathrm{g}$ trypsin inhibitor were added and the cell suspension was maintained in ice until required. A Silverson laboratory mixer equipped with a 'micro' head to provide the necessary shearing action was used to disrupt the spermatozoa. The cells were subjected to $25 \mathrm{sec}$ homogenization using half maximal speed, and they were maintained in ice throughout the procedure.

Discontinuous sucrose density gradients were used to isolate the plasma membrane fraction; these consisted of $2 \mathrm{ml}$ layers of $50 \%, 40 \%$ and $30 \%$ sucrose in $10 \mathrm{ml}$ polycarbonate centrifuge tubes. Approximately $2 \mathrm{ml}$ of the disrupted sperm suspension was placed at the top of each density gradient, followed by centrifugation at $68000 \mathrm{~g}$ for $1.5 \mathrm{~h}$ in an MSE $3 \times 25 \mathrm{ml}$ swing-out rotor. Membrane fractions were removed from the gradients using hand-held Pasteur pipettes.

\section{Lipid extraction}

The solvents used for the lipid extraction procedures contained $0.001 \%$ butylated hydroxytoluene as an antioxidant.

Whole spermatozoa. Approximately $1 \mathrm{ml} \mathrm{ram} \mathrm{semen} \mathrm{was} \mathrm{washed} \mathrm{twice,} \mathrm{using} \mathrm{a} \mathrm{ficoll} \mathrm{column} \mathrm{fol-}$ lowed by a Hepes-sucrose buffer, as described above. The spermatozoa were centrifuged to form a pellet, then lipids were extracted using chloroform and methanol $(2: 1 \mathrm{v} / \mathrm{v})$ as described by Christie (1973). The combined filtrates obtained from the extraction procedure were further purified by washing with saline (Folch, Lees \& Sloane-Stanley, 1957); after being allowed to separate overnight at $5^{\circ} \mathrm{C}$, the lower phase containing the lipids was removed and concentrated by evaporation under 
reduced pressure. The lipids were then redissolved in a small quantity of chloroform and stored under nitrogen at $-20^{\circ} \mathrm{C}$ until required.

Plasma membrane fraction. Aliquants of the plasma membrane fraction (band 2 ), containing $\sim 1$ mg protein, were ultracentrifuged. The membrane pellet was removed from the tube with $3.5 \mathrm{ml}$ methanol, then $7 \mathrm{ml}$ chloroform were added. The saline washing procedure was then carried out, and the lipids were collected and concentrated as described above.

\section{Separation of phospholipid classes by thin-layer chromatography (t.l.c.)}

The lipid extracts obtained from whole spermatozoa or membrane fractions were separated using $10 \times 10 \mathrm{~cm}$ high performance t.l.c. plates coated with silica gel (Nano-DC plates; Schleicher and Schüll, Dassel, FRG). The lipid extracts were applied as $1.5 \mathrm{~cm}$ bands, then developed in one dimension using chloroform :methanol : water $(60: 30: 4$ by vol.) (Williams, Davidson, Stevens \& Crawford, 1977) as the solvent system. Upon completion of development the lipid bands were located using iodine vapour.

Identification of the unknown lipid bands was accomplished by comparison of their mobilities with authentic reference phospholipids. The standard compounds, phosphatidylcholine, phosphatidylethanolamine, sphingomyelin, phosphatidylinositol and phosphatidylserine were purchased from Sigma; samples of cardiolipin were purchased from Sigma and from Applied Sciences Ltd (PA, U.S.A).

In addition, specific spray reagents were used for phospholipid identification. Molybdenum blue reagent (Sigma) was used to distinguish phosphorus-containing compounds; amino lipids, i.e. phosphatidylethanolamine and phosphatidylserine, were distinguished by the use of ninhydrin, and choline-containing lipids, i.e. choline phosphoglycerides and sphingomyelin, were detected using Dragendorff's reagent (Christie, 1973).

\section{Analytical methods}

Phospholipids were estimated by measurement of their phosphorus content. Bands of silica gel containing individual lipids were scraped from the t.l.c. plate into glass tubes. Lipid decomposition was then achieved using the method described by Bartlett (1959). After completion of the digestion procedure $3 \mathrm{ml}$ distilled water were added to each glass tube, and the silica gel was sedimented by centrifugation. Phosphorus content within the supernatant fluid was then assayed using a colorimetric procedure (kit supplied by Sigma) based on the Fiske \& SubbaRow (1925) technique.

Phosphorus content of unfractionated lipid extracts was estimated using the procedures described above; parallel cholesterol assays, for the detection of free and esterified cholesterol, were also performed using a ferric chloride technique (Rudel \& Morris, 1973), and the results were used to calculate cholesterol :phospholipid molar ratios.

\section{Electron microscopy}

For morphological studies the three membrane fractions were pelleted by further ultracentrifugation, then fixed for electron microscopy in a solution containing picric acid, formaldehyde and glutaraldehyde (Jones, 1973). Samples of the cellular debris were also fixed in this solution. After fixation, the membrane pellets and the debris were washed in sucrose/cacodylate buffer (Jones, 1973), osmicated in a $1: 1$ mixture of $2 \%$ osmium tetroxide and $3 \%$ potassium ferrocyanide (Russell \& Burguet, 1977), dehydrated using ethanol and embedded in araldite. Sections were either mounted on copper grids and stained in uranyl acetate and lead citrate (Venable \& Coggeshall, 1965), or mounted on gold grids and stained with $4 \%$ phosphotungstic acid (PTA).

In some separate experiments, the membranes and cellular debris were rapidly dehydrated in ascending grades of acetone, and then embedded in epon. Sections were mounted on gold grids, and stained using the chromic acid-PTA technique described by Clegg, Morré \& Lunstra (1975). 
Enzyme assays

Total protein was assayed using a modification of the Lowry procedure (microprotein determination kit Sigma) and inorganic phosphate was measured as described above.

Alkaline phosphatase activity was determined by measuring the hydrolysis of $p$-nitrophenol phosphate (kit supplied by Sigma). 5'-Nucleotidase activity was determined by measuring the amount of phosphate released after incubating $25-50 \mu \mathrm{g}$ membrane protein at $37^{\circ} \mathrm{C}$ in $1 \mathrm{ml} 50 \mathrm{mM}$ Tris buffer ( $\mathrm{pH} \mathrm{7.4)} \mathrm{containing} 2 \mathrm{~mm}$-adenosine monophosphate and $5 \mathrm{~mm}-\mathrm{MgCl}_{2}$. The reaction was stopped by the addition of ice-cold trichloroacetic acid.

ATPase in the membrane fraction was assayed using the procedure described above for $5^{\prime}$ nucleotidase, except that $2 \mathrm{~mm}$-adenosine triphosphate was included as the substrate (Rottem, Yashouv, Ne'eman \& Razin, 1973). The effects of sodium, potassium, magnesium and calcium ions upon ATPase activities were examined in a series of experiments (see Table 1); the results were analysed using the non-parametric Kruskal-Wallis analysis of variance (Conover, 1980).

Contamination of the membrane fraction by mitochondrial enzymes was assessed by measurement of succinate-cytochrome $\mathrm{C}$ reductase activity, as described by Ivanov \& Profirov (1981).

\section{Image analysis of electron micrographs}

Electron micrographs of the various membrane fractions were examined by semi-automatic image analysis (VIDS system: Analytical Measuring Systems Ltd, U.K.) to compare the mean vesicular diameters obtained from different regions of the density gradients. Mean vesicle diameter for each fraction was derived from cross-sectional area measurements of 50 vesicles.

In a separate series of measurements, electron micrographs of the cellular debris deposited during ultracentrifugation were examined by image analysis to determine the proportion of the available plasma membrane isolated by these procedures. The following procedure was used.

(a) The total length of sperm surface available, irrespective of the presence or absence of plasma membrane, was determined for each of 12 electron micrographs (original magnification $\times 5000$, enlarged $\times 2.5$ ). This was measured by outlining the perimeter of each feature with the pen of the graphics tablet and 40-60 individual features were measured on each micrograph.

(b) The total length of retained plasma membrane for each feature, together with the perimeters of the plasma membrane vesicles scattered amongst these sperm structures, were then measured in a similar way.

A simple ratio 'length of plasma membrane/length of available surface' was then calculated.

A similar series of measurements was performed in which the extent of acrosomal membrane disruption was examined. Sagittal sections through 25 acrosomes were examined; the total length of acrosomal membrane present, and bounding structurally intact regions of the acrosomal matrix, was measured using the graphics tablet. The outlines of this same series of acrosomes were then traced onto paper, and regions of missing membrane were drawn in by hand; the total perimeter

\section{PLATE 1}

Fig. 1. Electron micrograph showing the appearance of plasma membrane vesicles recovered from band 2. $\times 50000$.

Figs 2 \& 3. Sections of spermatozoa from the cellular debris deposited during ultracentrifugation. Figure 2 shows non-osmicated spermatozoa stained with $4 \%$ aqueous PTA. Sagittal sections of sperm heads are shown in both figures, and it is apparent from the conventionally stained preparation (Fig. 3) that most of the acrosomes have remained intact. This is confirmed in Fig. 2, in which PTA-positive material lines the inner acrosomal membrane (arrows) as in intact spermatozoa. Fig. 2, $\times 20000$; Fig. $3, \times 15750$. 
PLATE 1
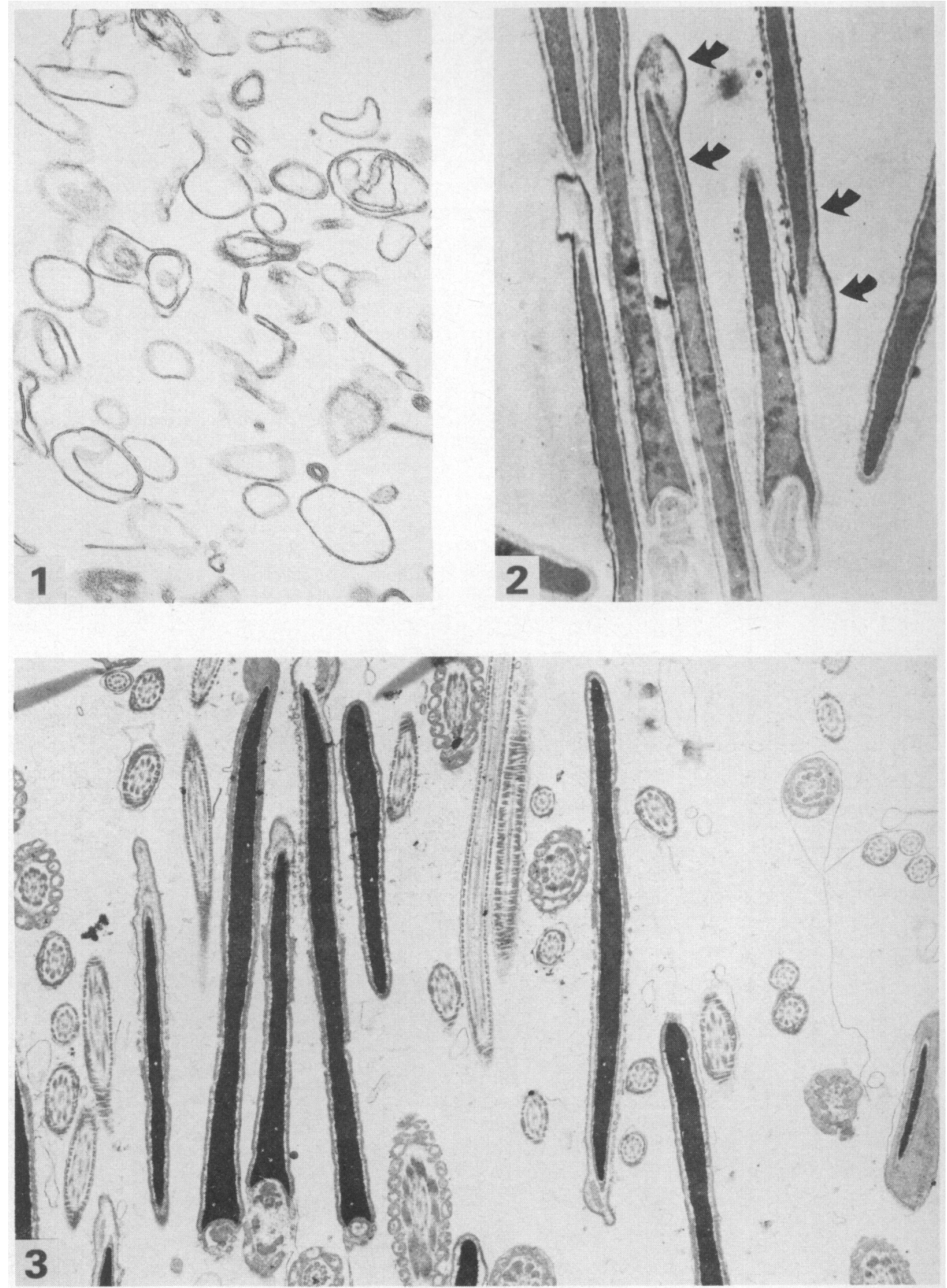
length of these artificially completed profiles was next measured to obtain a value representative of the maximum possible acrosomal membrane length.

A ratio of these two lengths, 'acrosomal membrane present:maximum possible acrosomal membrane length', was then calculated as an indicator of the proportion of acrosomal membrane remaining intact after the disruption and centrifugation steps.

\section{Results}

\section{Morphological aspects}

Membrane fractions were isolated at each of the three interfaces within the sucrose gradients. The band situated above the $30 \%$ sucrose layer (band 1) contained the least membrane, although vesicles were observed by electron microscopy.

The major membrane fraction (band 2) was isolated at the $40 / 30 \%$ sucrose interface. Most of the membrane observed by electron microscopy was in the form of smooth, empty, round or oval vesicles (mean radius $97 \pm 4.6 \mathrm{~nm}$ (s.e.m.), which were free of adherent material (Pl. 1, Fig. 1).

The membrane fraction which separated into the $50 / 40 \%$ sucrose interface (band 3 ) also contained round or oval vesicles. Their mean radius $( \pm$ s.e.m.) of $102 \pm 6.0 \mathrm{~nm}$ was not significantly different from that of the band 2 vesicles. Sheets of membrane were also occasionally observed, together with flattened vesicles and some particulate matter. Vesicles containing flocculent material were also apparent as a minor component of this fraction.

Mean protein concentrations ( \pm s.e.m.) for four different preparations of bands 2 and 3 were $0.438 \pm 0.076 \mathrm{mg} / \mathrm{ml}$ and $0.227 \pm 0.05 \mathrm{mg} / \mathrm{ml}$ respectively. Protein determinations were not performed on band 1 because of the presence of exogenous bovine serum albumin in the original sample.

The cellular debris deposited at the bottom of the sucrose gradients during centrifugation (Pl. 1, Fig. 3) contained whole spermatozoa stripped of their plasma membranes, but retaining their mitochondria and acrosomes. Morphological examination was sufficient to show that most of the acrosomes retained their contents, and were still encapsulated by the acrosomal membrane. The stereometric measurements demonstrated that $86 \pm 4 \%$ (s.e.m.) of the total acrosomal perimeter visible in 25 sagittal sections was occupied by undamaged outer acrosomal membrane. Although this figure seems relatively low $7 / 25$ acrosomal membranes were fully intact, and a further $8 / 25$ were in the range $90-99 \%$ intact. Of the 25 acrosomal sections, only 3 were significantly damaged, presenting $36 \%, 43 \%$ and $46 \%$ intact acrosomal membranes respectively. The remaining sections were all within the range $75-87 \%$ intact.

The use of $4 \%$ PTA as a specific stain for acrosomal material associated with the outer acrosomal membrane (Holt, 1979) demonstrated further that acrosomal membranes were not removed significantly by the procedures used (P1. 1, Fig. 2). Insufficient differentiation between the acrosomal and plasma membranes was obtained with the chromic acid/PTA technique for its successful application in this study.

Stereometric measurements of the cellular debris showed that the mean \pm s.e.m. ratio of 'plasma membrane present : available sperm surface' was $32 \cdot 7 \pm 2.5 \%$. Although this is a relatively crude way to measure the efficiency of the membrane isolation technique, it appears to indicate that $\sim 70 \%$ of available plasma membrane is removed from the original homogenate.

\section{Enzyme assays}

The membrane fraction recovered in band 2 showed both alkaline phosphatase $(10 \cdot 1 \pm 1 \cdot 74$ (s.e.m.) $\mu \mathrm{M}-\mathrm{Pi} / \mathrm{mg}$ protein $/ \mathrm{h}$ ) and $5^{\prime}$-nucleotidase activities $(0.27 \pm 0.09$ (s.e.m.) $\mu \mathrm{M}-\mathrm{Pi} / \mathrm{mg}$ protein $/ \mathrm{h}$ ). Succinate-cytochrome $\mathrm{C}$ reductase activity in this membrane fraction was negligible. 
Sodium-activated and calcium-activated ATPases were present (see Table 1); addition of these ions to the incubation mixtures caused significant increases in enzyme activity compared with treatments lacking added ions, or containing magnesium only $(P<0.025$ for both).

Table 1. Effects of ionic additions on ATPase activities in plasma membrane fractions from ram spermatozoa

\begin{tabular}{lcc}
\hline \multicolumn{1}{c}{ Additions $\dagger$} & $\begin{array}{c}\text { No. of } \\
\text { replicates }\end{array}$ & $\begin{array}{c}\text { Mean } \pm \text { s.e.m. } \\
\text { specific activities } \\
(\mu \mathrm{M} \cdot \mathrm{Pi} / \mathrm{mg} \text { protein/h) }\end{array}$ \\
\hline None & 4 & $8 \cdot 1 \pm 2 \cdot 4$ \\
$\mathrm{Mg}^{2+}$ & 6 & $12 \cdot 7 \pm 2 \cdot 4$ \\
$\mathrm{Mg}^{2+}, \mathrm{Na}^{+}$ & 3 & $29 \cdot 3 \pm 3 \cdot 5^{* *}$ \\
$\mathrm{Mg}^{2+}, \mathrm{Na}^{+}, \mathrm{K}^{+}$ & 3 & $19 \cdot 9 \pm 6 \cdot 0$ \\
$\mathrm{Ca}^{2+}$ & 4 & $25 \cdot 5 \pm 2 \cdot 8^{* *}$ \\
$\mathrm{Mg}^{2+}, \mathrm{Ca}^{2+}$ & 3 & $24 \cdot 3 \pm 3 \cdot 4^{* *}$ \\
\hline
\end{tabular}

$\dagger$ Assay conditions are described in 'Materials and Methods'. The salt concentrations used were $5 \mathrm{~mm}-\mathrm{MgCl}_{2}, 100 \mathrm{~mm}-\mathrm{NaCl}, 20 \mathrm{~mm}-\mathrm{KCl}, 5 \mathrm{~mm}-$ $\mathrm{CaCl}_{2}$.

\# Each replicate value is the mean of duplicate assays.

** Enzyme activities significantly higher $(P<0.025)$ than in the absence of sodium or calcium ions.

\section{Lipid analysis}

Four major classes of phospholipids, choline phosphoglycerides, ethanolamine phosphoglycerides, cardiolipin and sphingomyelin, were identified in whole ram spermatozoa, and their proportional occurrence is shown in Table 2. The plasmalogens and their respective ester-linked phospholipids were not distinguished in this study. The same 4 phospholipids were also identified in the lipid extracts prepared from the plasma membrane fractions (Table 2).

Table 2. Phospholipid composition* and cholesterol : phospholipid molar ratio of whole spermatozoa and plasma membrane fraction

\begin{tabular}{lcr}
\hline \multicolumn{1}{c}{ Phospholipid } & Whole spermatozoa $\dagger$ & Membrane $\ddagger$ \\
\hline Choline phosphoglycerides & $63.8 \pm 2.9$ & $65.23 \pm 3.41$ \\
Ethanolamine phosphoglycerides & $20.69 \pm 1.58$ & $12.44 \pm 1.73$ \\
Cardiolipin (diphosphatidylglycerol) & $4 \cdot 75 \pm 1.0$ & $2.59 \pm 0.55$ \\
Sphingomyelin & $11.15 \pm 1 \cdot 17$ & $19.77 \pm 1.47$ \\
Cholesterol : phospholipid ratio & $0.59 \pm 0.05$ & $\S 0.85 \pm 0.03$ \\
\hline
\end{tabular}

* Results are presented as percentages of total phospholipids recovered from t.l.c. plates.

$\dagger$ Means \pm s.e.m. of 4 replicates.

$\ddagger$ Means \pm s.e.m. of 6 replicates.

$\S$ Mean \pm s.e.m. of 5 replicates.

These results, which were analysed by a one-way analysis of variance and by the use of $t$ tests, showed that the isolated membrane fraction contained a significantly higher proportion of sphingomyelin than was present in the whole sperm lipid extract $(P<0.005)$, but conversely, a significantly lower proportion of phosphatidylethanolamine $(P<0.025)$.

Estimates of the cholesterol/phospholipid molar ratios for whole spermatozoa and isolated membranes are shown in Table 2 . The plasma membrane fraction possessed a significantly higher cholesterol content than the whole sperm lipid extract $(P<0 \cdot 01)$. 


\section{Determination of thermal phase-transition behaviour}

The occurrence of major thermal phase transitions amongst the lipid components of the membrane vesicles was examined by monitoring the initial velocity of the calcium-activated membrane ATPase, measured as inorganic phosphate released in $10 \mathrm{~min}$, as a function of temperature. As calcium-ATPase of the ram spermatozoon is an integral membrane protein (Bradley \& Forrester, 1980a), it is believed that these results show the existence of a thermal phase transition amongst the lipids in the immediate vicinity of the enzyme.

An Arrhenius plot of the data from one of 4 such experiments is shown in Text-fig. 1; a biphasic relationship between temperature and enzyme activity was apparent, with a change in slope, or discontinuity, at $22^{\circ} \mathrm{C}$. If the 4 replicates were plotted individually, each point representing the means of duplicate assays, then 4 similar graphs showing discontinuities were obtained. The mean ( \pm s.e.m.) of the 4 discontinuities obtained in this way was $23.8 \pm 0.82^{\circ} \mathrm{C}$.

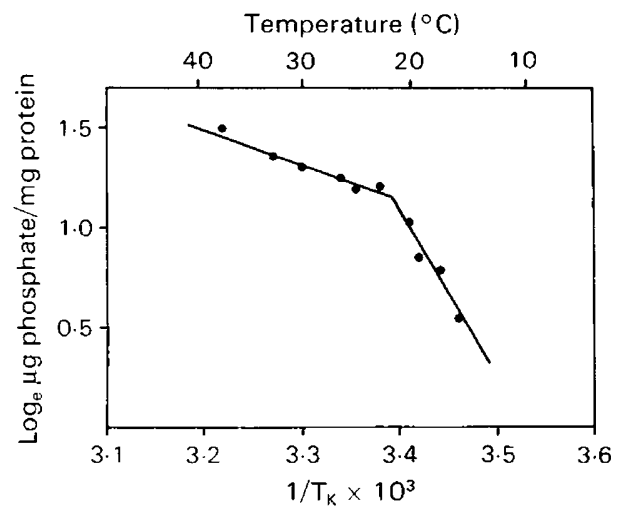

Text-fig. 1. Activity of calcium-stimulated ATPase in isolated sperm plasma membrane vesicles as a function of temperature. The graph is shown in the form of an Arrhenius plot, where the abscissa represents reciprocal absolute temperature $(\times 1000)$, and the ordinate shows the initial reaction velocity expressed as $(\log )$ micrograms phosphate released $/ \mathrm{mg}$ protein $/ \mathrm{min}$.

\section{Discussion}

The techniques presented in this paper for the isolation of plasma membranes from ram spermatozoa are relatively simple, but effective. The stereometric study of acrosomal disruption during the course of the isolation procedures showed that $80-90 \%$ of the acrosomal membrane remained undamaged; this figure would indicate a low level of contamination by outer acrosomal membrane fragments. Peterson, Russell, Bundman \& Freund (1980), whilst examining the efficiency of their own procedure for removing plasma membranes from boar spermatozoa, estimated that the plasma membrane covering the acrosome accounted for $34 \%$ of the total surface areas of a single cell. As boar and ram spermatozoa are of similar shape, this figure is probably approximately applicable to the ram spermatozoon. If this assumption is valid, it can be estimated from the stereometric results that only $3-7 \%$ contamination of the final membrane fraction by outer acrosomal membrane would occur. In broad agreement with this estimate, cytochemical examination of the membrane pellets failed to detect any of the PTA-positive material which characteristically lines the inner aspect of the outer acrosomal membrane (Holt, 1979). PTA-positive material occurred, however, in association with the acrosomal membranes of spermatozoa within the cellular debris, indicating the retention of most of the outer acrosomal membrane within this fraction. 
The membrane fraction recovered from band 2 in the present study was morphologically the most homogeneous, and was also the most concentrated both in terms of membrane yield and protein concentration. This fraction was therefore regarded as the most useful, and was used both for the enzyme determinations presented here and for the lipid analyses. The differences between this and the other major fraction (band 3) may be of potential interest, however, since it is believed that they both represent plasma membrane fractions. The separation of surface membranes into bands of two different densities was the subject of comment by Glick (1976), who argued that they might represent regions of different protein and glycoprotein composition; such membrane may be isolated from different regions of the sperm surface.

The patterns of enzyme activity reported in this paper agree in general with previous results. Ivanov \& Profirov (1981) reported the presence of a magnesium-stimulated sodium/potassium ATPase in the ram sperm plasma membrane, whilst Bradley \& Forrester $(1980 \mathrm{a}, \mathrm{b})$ and Breitbart, Stern \& Rubinstein (1983) have described calcium-stimulated ATPase. According to Bradley \& Forrester (1980a) the calcium-stimulated ATPase is found in the sperm tail, a finding confirmed for bovine spermatozoa by Vijayasarathy \& Balaram (1982), who reported a ratio of $40 / 3$ for the activities of this enzyme in sperm tails and heads respectively.

Whilst the specific activities reported here for the ATPases under various conditions are comparable to those in other studies, the alkaline phosphatase activity was 5-fold higher than that reported by Ivanov \& Profirov (1981), and the 5 '-nucleotidase activity was about 30 -fold lower. The reasons for these differences are unclear, although they may reflect differences in incubation conditions.

The observation that the molar ratio of cholesterol to phospholipid of the ram sperm plasma membrane was in the region of $85 \%$ is in keeping with estimates for plasma membranes from other sources (for reviews, see Glick, 1976; Thompson, 1980). The lower ratio (59\%) obtained for lipid extracts from whole spermatozoa is somewhat higher than the figure of $38 \%$ previously reported by Darin-Bennett \& White (1977), obtained by the same assay procedure, but emphasizes that analysis of the whole spermatozoon consistently underestimates the sterol content. Rabbit and human spermatozoa, therefore, with whole sperm cholesterol : phospholipid ratios of $88 \%$ and $99 \%$ (DarinBennett \& White, 1977), respectively, might possess plasma membranes with unusually high concentrations of sterol. Comparative studies of sperm membrane composition might therefore be especially profitable, both from a physiological viewpoint and in relation to stability during thermal and osmotic stress, bearing in mind that cholesterol is an effective modulator of membrane fluidity, and hence also stability.

The examination of thermal phase-transition behaviour in the isolated membrane fraction by the measurement of enzyme activity at various temperatures is a well established procedure which has been applied to a number of systems in animals, micro-organisms and plants (for reviews, see Kimelberg, 1977; Quinn, 1981; Chapman, 1982). The results obtained in this study indicate that the lipids in the immediate vicinity of the calcium-stimulated ATPase molecule show a major thermal phase transition, evidenced by a change in the activation energy of the enzyme reaction, in the region of $23-24^{\circ} \mathrm{C}$. As the ram spermatozoon is an example of a cold-shock susceptible cell it would be of interest to compare this result with values obtained from human and rabbit spermatozoa, which are more resistant to cold shock.

The percentage distribution of the major phospholipids in whole spermatozoa reported here is in good agreement with values shown by Scott (1973) and Darin-Bennett, Poulos \& White (1976). However, the differences in phospholipid composition between whole spermatozoa and isolated plasma membranes are difficult to interpret at this stage, although the importance may become clearer through comparative studies. The heterogeneity of the sperm membrane system demands, however, that studies using precise methods of localizing these lipids are required before their importance can be considered. Nevertheless, in a comparison of lymphoid cells and their respective plasma membranes, higher levels of sphingomyelin, and lower levels of phosphatidylethanolamine, were found amongst the membrane lipids (Koizumi et al., 1981), as reported in the present study.

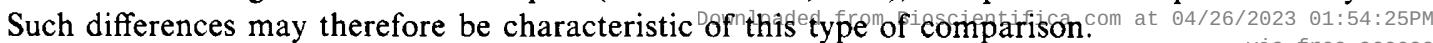


A small (2.6\%) amount of cardiolipin was detected amongst the phospholipids of the plasma membrane fraction. Although this lipid occurs predominantly within mitochondria, it has been reported to be a minor plasma membrane component of a variety of cells (Ioannou \& Golding, 1979). Bearer \& Friend (1982) detected the incorporation of labelled phosphate into the cardiolipin of guinea-pig spermatozoa during incubation in Tyrode's solution, and suggested that this anionic lipid might actively promote cell fusion events during fertilization. Studies aimed at localizing cardiolipin in the sperm plasma membrane might therefore increase understanding of the molecular events which accompany fertilization.

We thank Dr P. F. Watson (Royal Veterinary College, London) for providing the samples of ram semen. The electron microscope used for this work was purchased with a grant from Elf-Aquitaine (U.K.), and the study was supported by a grant from the Agricultural and Food Research Council.

\section{References}

Bartlett, G.H. (1959) Phosphorus assay in column chromatography. J. biol. Chem. 234, 466-468.

Bearer, E.L. \& Friend, D.S. (1982) Modifications of anionic-lipid domains preceding membrane fusion in guinea pig sperm. J. Cell Biol. 92, 604-615.

Bradley, M.P. \& Forrester, I.T. (1980a) A $\left\{\mathrm{Ca}^{2+}+\right.$ $\left.\mathrm{Mg}^{2+}\right\}-A T P a s e$ in the plasma membranes isolated from ram sperm flagella Cell Calcium 1, 381-390.

Bradley, M.P. \& Forrester, I.T. (1980b) The isolation of plasma membranes from ram sperm flagella. Proc. Univ. Otago Med. Sch. 58, 3-4.

Breitbart, H., Stern, B. \& Rubinstein, S. (1983) Calcium transport and $\mathrm{Ca}^{2+}$-ATPase activity in ram spermatozoa plasma membrane vesicles. Biochim. Biophys. Acta 728, $349-355$.

Chapman, D. (1982) Protein-lipid interactions in model and natural biomembranes. In Biological Membranes, pp. 179-229. Ed. D. Chapman. Academic Press, London.

Christie, W.W. (1973) Lipid Analysis. Pergamon Press, Oxford.

Clegg, E.D., Morré, D.J. \& Lunstra, D.D. (1975) Porcine sperm membranes: in vivo phospholipid changes, isolation and electron microscopy. In The Biology of the Male Gamete, pp. 321-335. Eds J. G. Duckett \& P. A. Racey. Academic Press, London.

Conover, W.J. (1980) Practical Non-parametric Statistics, 2nd edn. Wiley, New York.

Darin-Bennett, A. \& White, I.G. (1977) Influence of cholesterol content of mammalian spermatozoa on susceptibility to cold-shock. Cryobiology 14, 466-470.

Darin-Bennett, A., Poulos, A. \& White, I.G. (1976) The fatty acid composition of the major phosphoglycerides of ram and human spermatozoa. Andrologia 8, 37-45.

Fiske, C.H. \& SubbaRow, Y. (1925) The colorimetric determination of phosphorus. J. biol. Chem. 66, 375400.

Folch, J., Lees, M. \& Sloane-Stanley, G.H. (1957) A simple method for the isolation and purification of total lipids from animal tissues. J. biol. Chem. 226, 497-509.

Glick, M.C. (1976) Isolation of surface membranes from mammalian cells. In Mammalian Cell Membranes, Vol. 1, pp. 45-77. Eds G. A. Jamieson \& D. M. Robinson. Butterworth, London.
Harrison, R.A.P. (1976) A highly efficient method for washing mammalian spermatozoa. J. Reprod. Fert. 48, 347-353.

Holt, W.V. (1979) Development and maturation of the mammalian acrosome: a cytochemical study using phosphotungstic acid staining. J. Ultrastruct. Res. 68, $58-71$.

Ioannou, P.V. \& Golding, B.T. (1979) Cardiolipins; their chemistry and biochemistry. Prog. Lipid Res. 17, 279318 .

Ivanov, N. \& Profirov, Y. (1981) Isolation of plasma membranes from ram spermatozoa by a two-phase polymer system. J. Reprod. Fert. 63, 25-29.

Jones, R.C. (1973) Preparation of spermatozoa for electron and light microscopy. J. Reprod. Fert. 33, $145-149$.

Kimelberg, H.K. (1977) The influence of membrane fluidity on the activity of membrane-bound enzymes. In Cell Surface Reviews, vol. 3, pp. 205-293. Eds G. Poste \& G. L. Nicolson. Elsevier/North-Holland, Amsterdam.

Koizumi, K., Shimizu, S., Koizumi, K.T., Nishida, K., Sato, C., Ota, K. \& Yamanaka, N. (1981) Rapid isolation and lipid characterization of plasma membranes from normal and malignant lymphoid cells of mouse. Biochim. Biophys. Acta 649, 393-403.

Peterson, R., Russell, L., Bundman, D. \& Freund, M. (1980) Evaluation of the purity of boar sperm plasma membranes prepared by nitrogen cavitation. Biol. Reprod. 23, 637-645.

Quinn, P.J. (1981) the fluidity of cell membranes and its regulation. Prog. Biophys. molec. Biol. 38, 1-104.

Rottem, S., Yashouv, J., Ne'eman, Z. \& Razin, S. (1973) Cholesterol in mycoplasma membranes. Composition, ultrastructure and biological properties of membranes from Mycoplasma mycoides var. capri cells adapted to grow with low cholesterol concentrations. Biochim. Biophys. Acta 323, 495508 .

Rudel, L.L. \& Morris, M.D. (1973) Determination of cholesterol using O-phthalaldehyde. J. Lipid Res. 14, 364-366.

Russell, L.D. \& Burguet, S. (1977) Ultrastructure of Leydig cells as revealed by secondary tissue treatment with a ferrocyanide-osmium mixture. Tissue \& Cell 9, $751-766$. 
Scott, T.W. (1973) Lipid metabolism of spermatozoa. $J$. Reprod. Fert., Suppl. 18, 65-76.

Thompson, G.A., Jr (1980) The Regulation of Membrane Lipid Metabolism. CRC Press, Baton Rouge.

Venable, J.H. \& Coggeshall, R. (1965) A simplified lead citrate stain for use in electron microscopy. $J$. Cell Biol. 25, 407-408.

Vijayasarathy, S. \& Balaram, P. (1982) Regional differentiation in bull sperm plasma membranes. Biochem. Biophys. Res. Commun. 108, 760-769.
White, I.G. \& Darin-Bennett, A. (1976) The lipids of sperm in relation to cold shock. Proc. 8 th Int. Congr. Anim. Reprod. \& A.I. Krakow, pp. 951-954.

Williams, G., Davidson, B.C., Stevens, P. \& Crawford, M.A. (1977) Comparative fatty acids of the dolphin and the herring. J. Am. Oil Chemists' Soc. 54, 328330 .

Received 31 May 1984 